\title{
Research on frequency correction method of broadband AC RMS converter
}

\author{
Wu Zhu, Bing-Yao Liu, and Jia-Min Zhang \\ Shanghai University of Electric Power, shanghai, China \\ Email:1257804918@qq.com
}

\begin{abstract}
In order to reduce the nonlinear error, a new method of nonlinear error correction in a wide frequency range is presented. In this method, firstly, the frequency of the measurement signal is divided into three bands. In each frequency band, linear fitting is used to pretreat the difference between the measured value and the output value of the standard source, and then the pretreatment is calibrated by using least square method in the whole frequency range. The correction results show that when the measured signal frequency is changed between $50 \mathrm{~Hz}$ and $30 \mathrm{kHz}$. After the frequency correction, the linearity of the measurement can reach $0.018 \%$. The correction effect of this method is better than the traditional least square method and the measurement accuracy of the broad band signal is improved.
\end{abstract}

Keywords: True RMS Converter; Error Correction; Piecewise Linear Fitting; Least Square Method.

\section{Introduction}

With the development of the power electronics and electrified railway, improvement of aviation metrology requirements, the application of high power frequency conversion speeder, etc, the frequency band of the precision test signal is becoming wider and wider, so it is very important to measure the wide bandwidth accurately. As the base of alternating current power measurement , the effective value of AC voltage can usually be characterized by using thermoelectric meter and arithmetic meter. Although the precision of the thermoelectric conversion is high and the bandwidth is wide, this method is very sensitive to the temperature of the environment .Besides, the complexity and the poor user-friendliness in the operation limit its widespread use. Considering the accuracy, stability, frequency band-width, linearity, manufacturing costs, etc, $\log$-antilog arithmetic circuit is usually used to realize the AC-DC true effective value conversion. In the measurements of broadband $\mathrm{AC}$ signals, due to the influence of distributing parameters on the input circuit, the attenuation coefficient varies with frequency, the limited of amplifier bandwidth and open 
loop gain, operation resistance distribution capacitance and the influence of the natural characteristic of $\log$-antilog operation circuit natural characteristic ,the output linearity of the log-antilog operation formula AC and DC converter varies with frequency. In order to improve the measurement accuracy of broadband signals, the output of the converter must be calibrated by the frequency nonlinear error correction[1].

For the measurement of single frequency AC signal, there are many nonlinear error calibration methods, such as parabola fitting method, piecewise broken line method, neural network method ${ }^{[2]}$, improved genetic algorithm ${ }^{[3]}$, support vector machine method ${ }^{[4]}$,etc. and the result is good. For wideband signal measurement, although the precision of the method proposed in the paper[5]can reach $0.06 \%$ in the wide frequency range from $50 \mathrm{~Hz}$ to $30 \mathrm{kHz}$, it still can not meet the requirement of the measurement accuracy of wide band signal. In order to further improve the measurement accuracy, a new method combining the fitting frequency segment line and the least square method is presented, which reduces the nonlinear measurement.

\section{The Basic Principle of Error Correction for Wideband Converter}

The nonlinear error of the logarithmic-antilogarithmic AC-DC converter mainly includes the component natural characteristic error and the circuit design conversion principle error, and the frequency correction must be carried out to improve the measurement accuracy of the wide band signal. The experimental results show that the nonlinear error caused by the frequency variation is significantly increased when the measured frequency bands becomes wider. In this paper, the correction process consists of two parts. The first part is precorrection which is based on the measured results to preliminary decreases nonlinear error. The second part is using the least square method[6][7]to calibrate the nonlinear error on the precorrected results within the measurement the range broadband signal. The calibration principle block diagram is shown in Figure 1, where $x$ is the input signal, $f$ is the signal frequency, is the output of RMS converter, $\Delta y$ is the difference between the output value of standard source and the measured value, $y^{\prime}$ is the precorrected result, and $\hat{y}$ is the final corrected output value. 


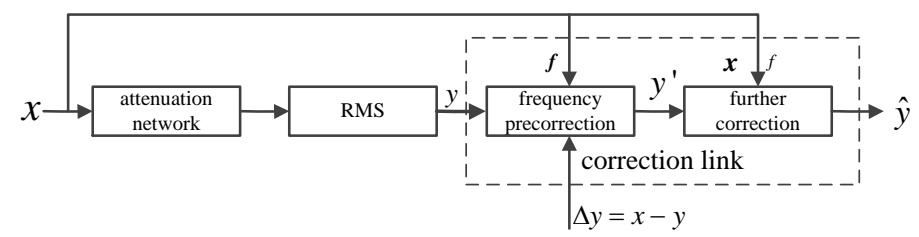

Fig. 1 schematic diagram of error correction

$$
y=f(x)
$$

In real application , $f(x)$ in the above Eq. (1) is an unknown nonlinear function, and certain nonlinear errors exist in the conversion results. In order to reduce the gain error and nonlinear error of the AC-DC converter, the output $y$ of the converter can be corrected through a correction link, and the corrected output value can be closer to the input value. The correction link is composed of two parts, the frequency precorrection and the further correction. In Fig.1, $y^{\prime}$ is a function of $y, \Delta y$, and $f$. The output expression of the frequency precorrection is the formula (2), $\hat{y}$ is a function of $y^{\prime}, x$ and $f$ where the corrected output expression is shown in Eq.(3).

$$
\begin{gathered}
y^{\prime}=F(y, \Delta y, f) \\
\hat{y}=F\left(y^{\prime}, x, f\right)
\end{gathered}
$$

\subsection{The principle of the frequency of precorrection}

In the test of $\mathrm{AC}$ parameters, the frequency band of the measured signal is wide. In order to improve the quality of nonlinear correction, the measurement frequency band is divided into three bands. Three steps are taken in the precorrection. Firstly, the function $R_{i p}(\Delta y, f)$ is obtained by using piecewise line fitting of $\Delta y$ with the change of $f$. Then the function $G(y, f)$ is obtained by using the least square method to fit $y$ with $f$. At last, the two fitting results are added together to get the precorrection function $F(y, \Delta y, f)$. The detailed correction method is as fellows: We assume that the frequency range of the measured signal is from $f_{1}$ to $f_{m}$ and its amplitude range is from $x_{0}$ to $\mathrm{x}_{n}$. The frequency of measured signal is divided into three bands: $f_{1} \sim f_{j 1}, f_{j 1} \sim f_{j 2}$, $f_{j 2} \sim f_{m}$. And then, linear fitting is performed in each frequency band. When the input signal is $x_{i}$, the precorrection function is:

$$
\mathrm{y}_{i}^{\prime}=G_{i}(y, f)+R_{i p}(\Delta y, f)
$$


In Eq.(4) $G_{i}(y, f)$ is the output value of the polynomial by using the least square method when the input is $x_{i}, f$ is the input signal frequency, $R_{i p}(\Delta y, f)$ is the linear fitting function in each frequency band. According to the principle of least square method, when the voltage is $x_{i}$, the coefficient of the polynomial $G_{i}(y, f)$ is :

$$
\left(\begin{array}{l}
a_{i 0} \\
a_{i 1} \\
\vdots \\
a_{i n}
\end{array}\right)=\left(\begin{array}{llll}
m & \sum_{j=1}^{m} f_{j} & \ldots & \sum_{j=1}^{m} f_{j}{ }^{n} \\
\sum_{j=1}^{m} f_{j} & \sum_{j=1}^{m} f_{j}^{2} & \ldots & \sum_{j=1}^{m} f_{j}^{n+1} \\
\vdots & \vdots & & \vdots \\
\sum_{j=1}^{m} f_{j}{ }^{n} & \sum_{j=1}^{m} f_{j}{ }^{n+1} & \ldots . . & \sum_{j=1}^{m} f_{j}^{n+1}
\end{array}\right)^{-1}\left(\begin{array}{c}
\sum_{j=1}^{m} y_{i j} \\
\sum_{j=1}^{m} f_{j} y_{i j} \\
\vdots \\
\sum_{i=1}^{m} f_{j}{ }^{m} y_{i j}
\end{array}\right)
$$

In Eq.(5), $f_{j}(j=1,2 \ldots m)$ is the frequency between $50 \mathrm{~Hz}-30 \mathrm{kHz}$ range , and $y_{i j}$ is the output at the input voltage $x_{i}$, and the frequency $f_{j}$. The linear fitting function in the three frequency bands are:

$$
\begin{gathered}
R_{i p}(\Delta y, f)=k_{i p} \cdot f+b_{i p} \\
\left\{\begin{array}{l}
R_{i 1}(\Delta y, f)=k_{i 1} \cdot f+b_{i 1}\left(f_{1} \leq f \leq f_{j 1}\right) \\
R_{i 2}(\Delta y, f)=k_{i 2} \cdot f+b_{i 2}\left(f_{j 1} \leq f \leq f_{j 2}\right)
\end{array}\right.
\end{gathered}
$$

In Eq.(6), $k_{i p}$ is a linear fitting term coefficient, $b_{i p}$ is a constant and $p$ is the serial number of the bands. In the three bands, $t$ frequency points are selected as the frequency correction reference points. The coefficient of the linear fitting function in the first band is:

$$
\left\{\begin{array}{l}
k_{i 1}=\left[t \cdot \sum_{j=1}^{t} f_{j} \cdot \Delta y_{i j}-\left(\sum_{j=1}^{t} f_{j} \sum_{j=1}^{t} \Delta y_{i j}\right)\right] /\left[t \cdot \sum_{j=1}^{t} f_{j}{ }^{2}-\left(\sum_{j=1}^{t} f_{j}\right)^{2}\right] \\
b_{i 1}=\left(\sum_{j=1}^{t} \Delta y_{i j}\right) / \mathrm{t}-k_{i 1} \cdot\left(\sum_{j=1}^{t} f_{j}\right) / \mathrm{t}
\end{array}\right.
$$

The linear fitting expression in the first band is then:

$$
\begin{aligned}
R_{i 1}(\Delta y, f)= & {\left[t \cdot \sum_{j=1}^{t} f_{j} \cdot \Delta y_{i j}-\left(\sum_{j=1}^{t} f_{j} \sum_{j=1}^{t} \Delta y_{i j}\right)\right],\left[t \cdot \sum_{j=1}^{t} f_{j}^{2}-\left(\sum_{j=1}^{t} f_{j}\right)^{2}\right] \cdot f+} \\
& \left(\sum_{j=1}^{t} \Delta y_{i j}\right) / \mathrm{t}-k_{i 1} \cdot\left(\sum_{j=1}^{t} f_{j}\right) / \mathrm{t}
\end{aligned}
$$

When the input voltage is $x_{i}$, the precorrection function is:

$$
\mathrm{y}_{i}^{\prime}=\left(a_{i n} \cdot f^{n}+a_{i n-1} \cdot f^{n-1}+\ldots . a_{i 1} \cdot f+a_{i 0}\right)+ \begin{cases}k_{i 1} \cdot f+b_{i 1} & \left(f_{1} \leq f \leq f_{j 1}\right) \\ k_{i 2} \cdot f+b_{i 2} & \left(f_{j 1} \leq f \leq f_{j 2}\right) \\ k_{i 3} \cdot f+b_{i 3} & \left(f_{j 2} \leq f \leq f_{m}\right)\end{cases}
$$


According to Eqs.(5),(7)and(9), error precorrection can be realized. And then the final frequency correction can be obtained by using the least squares method.

\subsection{Further correction principle}

Because only the nonlinear error caused by frequency band broadening is corrected in the the precorrection, and the input voltage across the range remains uncorrected, the precorrection can not meet the requirements of the measurement accuracy of wide band signal. Therefore, further correction should be carried on the precorrection result. In further correction, the results of the precorrection are fitted by using the least square method to reduce the error. The detailed steps are: firstly, $m$ frequency correction reference points labeled as $f_{1}, f_{2} \ldots . f_{m}$ are selected in the whole frequency range, at certain frequency $f_{j}$ $(j=1,2 \ldots m), n+1$ voltage correction reference points are selected, with n-order polynomial fitting. Assume that the input value is $x_{i}(i=0,1,2 \ldots n)$, the frequency is $f_{j}$, the corresponding output value is $\mathrm{y}_{i j}^{\prime}(i=0,1,2 \ldots n, j=1,2 \ldots m)$, the coefficient of n-order polynomial is $b_{i s}(i=0,1 \ldots n, s=0,1 \ldots n)$. According to the principle of least square method, when the input signal frequency is $f_{j}$, the coefficient of calibration polynomial is:

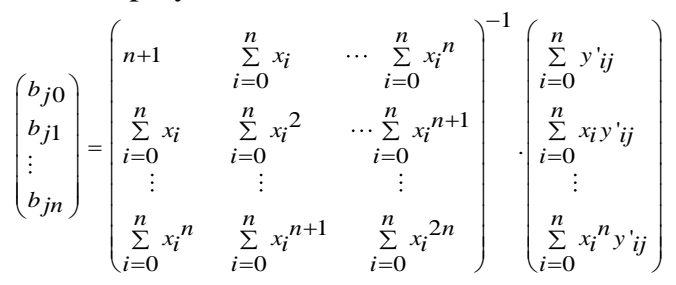

When frequency changes at different frequency reference points, polynomial coefficient $b_{i s}$ varies with frequency, so the calibration polynomial coefficient is a function of frequency. Therefore, it is necessary to carry out the polynomial fitting of the frequency again in the measurement frequency range. Assumed that the polynomial of an arbitary coefficient is $b_{j q}(j=1,2 \ldots m$; $q=0,1,2 . . n$ ) which is about the frequency of polynomial and the polynomial coefficient is $c_{q j}(q=0,1,2 \ldots n, j=0,1 \ldots m-1)$, the polynomial coefficients can be written as follows: 


$$
\left(\begin{array}{l}
c_{j 0} \\
c_{j 1} \\
\vdots \\
c_{j m-1}
\end{array}\right)=\left(\begin{array}{llll}
m & \sum_{j=1}^{m} f_{j} & \cdots & \sum_{j=1}^{m} f_{j}{ }^{m} \\
\sum_{j=1}^{m} f_{j} & \sum_{j=1}^{m} f_{j}^{2} & \ldots & \sum_{j=1}^{m} f_{j}^{m+1} \\
\vdots & \vdots & & \vdots \\
\sum_{j=1}^{m} f_{j}{ }^{m} & \sum_{j=1}^{m} f_{j}^{m+1} \ldots & \sum_{j=1}^{m} f_{j}^{2 m}
\end{array}\right)^{2 m} \cdot\left(\begin{array}{l}
\sum_{j=1}^{m} b_{1 q} \\
\sum_{j=1}^{m} f_{j} b_{2 q} \\
\vdots \\
\sum_{j=1}^{m} f_{j}{ }^{m} b_{m q}
\end{array}\right)
$$

When the voltage and frequency are changed, the correction output value is:

$$
\begin{aligned}
& \hat{y}=\left(c_{n n} \cdot f_{j}{ }^{n}+c_{n n-1} \cdot f_{j}{ }^{n-1}+\ldots+c_{n 1} \cdot f_{j}{ }^{1}+c_{n 0}\right) \cdot x^{n}+\left(c_{n-1 n} \cdot f_{j}{ }^{n}+c_{n-1 n-1} \cdot f_{j}{ }^{n-1}+\right. \\
& \left.\ldots+c_{n-11} \cdot f_{j}{ }^{1}+c_{n-10}\right) \cdot x^{n-1} \ldots \ldots\left(c_{1 n} \cdot f_{j}{ }^{n}+c_{1 n-1} \cdot f_{j}{ }^{n-1}+\ldots+c_{11} \cdot f_{j}{ }^{1}+c_{10}\right) \cdot x+\left(c_{0 n}\right. \\
& \left.\cdot f_{j}{ }^{n}+c_{0 n-1} \cdot f_{j}{ }^{n-1}+\ldots+c_{01} \cdot f_{j}{ }^{1}+c_{00}\right)
\end{aligned}
$$

\section{Calibration Experiment of Wide Band Data}

The AC voltage measurement setup ${ }^{[8]}$ is studied to verify the validity of this met hod. The input frequency is in the range of $50 \mathrm{~Hz}$ to $30 \mathrm{kHz}$.In the experimental $\mathrm{d}$ ata preprocessing based on Eqs. (9) and (12), $1500 \mathrm{~Hz}, 2000 \mathrm{~Hz}, 4000 \mathrm{~Hz}, 6000 \mathrm{H}$ $\mathrm{z}, 9000 \mathrm{~Hz}, 12000 \mathrm{~Hz}, 17000 \mathrm{~Hz}, 25000 \mathrm{~Hz}, 30000 \mathrm{~Hz}$ are selected as frequency po ints for fitting. According to Eq.(5), when the voltage range is $150 \mathrm{~V}$ and the inp ut voltage is $1 \mathrm{~V}$, the quadratic trinomial coefficients labeled as $\mathrm{a}_{0}(1), \mathrm{a}_{1}(1)$ and $\mathrm{a}_{2}$ (1) are fitted; when the input voltage is $80 \mathrm{~V}$, the quadratic trinomial coefficients labeled as $a_{0}(80), a_{1}(80)$ and $a_{2}(80)$ are fitted; when the input voltage is $150 \mathrm{~V}$, qu adratic trinomial coefficients labeled as $\mathrm{a}_{0}(150), \mathrm{a}_{1}(150), \mathrm{a}_{2}(150)$ are fitted. The $\mathrm{ab}$ ove coefficients are shown in Tab. 1.

Tab. 1 Quadratic trinomial coefficient

\begin{tabular}{cccc}
\hline coefficient/voltage $(\mathrm{V})$ & 1 & 80 & 150 \\
\hline $\mathrm{a}_{2}$ & $1.2265074 * 10^{-11}$ & $2.17162137 * 10^{-10}$ & $2.2755290^{*} 10^{-10}$ \\
$\mathrm{a}_{1}$ & $-1.9004365 * 10^{-6}$ & $-1.6868966^{*} 10^{-5}$ & $-2.7711339 * 10^{-5}$ \\
$\mathrm{a}_{0}$ & $9.9186573 * 10^{-1}$ & $7.9937467 * 10^{+1}$ & $1.49847807 * 10^{+2}$ \\
\hline
\end{tabular}

From Tab. 1, when the input voltage is $1 \mathrm{~V}$, the function is:

$$
G_{1}(y, f)=1.2265074 \times 10^{-11} f^{2}-1.9004365 \times 10^{-6} \cdot f+9.9186573 \times 10^{-1}
$$

Similarly, $G_{80}(y, f)$ and $G_{150}(y, f)$ can also be deduced from Table 1.

The correction band $50 \mathrm{~Hz} \sim 30 \mathrm{kHz}$ is divided into three frequency subands:50Hz $\sim \mathrm{kHz}, 9 \mathrm{KHz} \sim 17 \mathrm{kHz}$ and $17 \mathrm{kHz} \sim 30 \mathrm{kHz}$. According to Eq.(7), the fitted linear coefficients can be obtained.

When the input value are $1 \mathrm{~V}, 80 \mathrm{~V}$ and $150 \mathrm{~V}$, the precorrection output are: 


$$
\begin{aligned}
& y_{1}^{\prime}=1.2265074 \times 10^{-11} \cdot f^{2}-1.9004365 \times 10^{-6} \cdot f+9.9186573 \times 10^{-1}+\left\{\begin{array}{l}
1.4661355 \times 10^{-6} \cdot f+9.4940239 \times 10^{-3} \quad(50 \mathrm{~Hz} \leq f \leq 9 \mathrm{kHz}) \\
3.0097087 \times 10^{-6} \cdot f-8.3203883 \times 10^{-3}(9 \mathrm{kHz} \leq f \leq 17 \mathrm{kHz}) \\
9.1836735 \times 10^{-7} \cdot f+2.4877551 \times 10^{-2}
\end{array}(17 \mathrm{kHz} \leq f \leq 30 \mathrm{kHz})\right. \\
& y_{80}^{\prime}=2.17162137 \times 10^{-10} \cdot f^{2}-1.6868966 \times 10^{-5} \cdot f+7.993746710^{+1}+\left\{\begin{array}{l}
1.3960159 \times 10^{-5} \cdot f+6.8051793 \times 10^{-3} \quad(50 \mathrm{~Hz} \leq f \leq 9 \mathrm{kHz}) \\
1.2893204 \times 10^{-5} \cdot f+7.9524272 \times 10^{-2} \quad(9 \mathrm{kHz} \leq f \leq 17 \mathrm{kHz}) \\
7.244898 \times 10^{-6} \cdot f+1.6086735 \times 10^{-1}
\end{array}(17 \mathrm{kHz} \leq f \leq 30 \mathrm{kHz})\right. \\
& y_{150}^{\prime}=2.2755290 \times 10^{-10} \cdot f^{2}-2.7711339 \times 10^{-5} \cdot f+1.49847807 \times 10^{2}+\left\{\begin{array}{l}
3.2557769 \times 10^{-5} \cdot f+1.282749 \times 10^{-1} \quad(50 \mathrm{~Hz} \leq f \leq 9 \mathrm{kHz}) \\
1.4242718 \times 10^{-5} \cdot f+2.7999029 \times 10^{-1} \quad(9 \mathrm{kHz} \leq f \leq 17 \mathrm{kHz}) \\
2.244898 \times 10^{-5} \cdot f+1.3267347 \times 10^{-1} \quad(17 \mathrm{kHz} \leq f \leq 30 \mathrm{kHz})
\end{array}\right.
\end{aligned}
$$

The frequency precorrection is actually the preprocessing for the experimen tal data. The least square method is used for the further frequency error correctio $\mathrm{n}$, Therefore, by putting the frequencies of $1500 \mathrm{~Hz}, 14 \mathrm{kHz}, 30 \mathrm{kHz}$ into Eqs. (14), (15) and (16),experimental data of at $(1 \mathrm{~V}, 1500 \mathrm{~Hz}),(1 \mathrm{~V}, 14 \mathrm{kHz}),(1 \mathrm{~V}, 30 \mathrm{kHz}),(80$ $\mathrm{V}, 1500 \mathrm{~Hz}),(80 \mathrm{~V}, 14 \mathrm{kHz}),(80 \mathrm{~V}, 30 \mathrm{kHz}),(150 \mathrm{~V}, 1500 \mathrm{~Hz})$ and $(150 \mathrm{~V}, 14 \mathrm{kHz})$ are pre processed .The results are shown in Tab. 2.

Tab. 2 Data preprocessing results

\begin{tabular}{cccc}
\hline voltage $(\mathrm{V}) /$ frequency $(\mathrm{Hz})$ & 1500 & 14000 & 30000 \\
\hline 1 & 1.001 & 1.001 & 0.998 \\
80 & 80.002 & 80.004 & 80.005 \\
150 & 149.984 & 149.984 & 150.027 \\
\hline
\end{tabular}

When the input frequency is $1500 \mathrm{~Hz}$, the fitting quadratic trinomial coefficients are $b_{0}(1500), b_{1}(1500)$ and $b_{2}(1500)$; when the input frequency is $14 \mathrm{kHz}$, the fitting quadratic trinomial coefficients are $\mathrm{b}_{0}(14 \mathrm{k}), \mathrm{b}_{1}(14 \mathrm{k})$ and $\mathrm{b}_{2}(14 \mathrm{k})$; when the input frequency is $30 \mathrm{kH}$, the fitting quadratic trinomial coefficients are $b_{0}(30 k), b_{1}(30 k)$ and $b_{2}(30 k)$. From Table 2, quadratic trinomial coefficients of corrected frequency reference point at the $15000 \mathrm{~Hz}, 14 \mathrm{kHz}$, $30 \mathrm{kHz}$ can be fitted, and the resulted are listed in Tab. 3 .

Tab. 3 Polynomial coefficients

\begin{tabular}{cccc}
\hline coefficient/frequency & $1500 \mathrm{~Hz}$ & $14 \mathrm{KHz}$ & $30 \mathrm{KHz}$ \\
\hline $\mathrm{b}_{2}$ & $-1.7818614 * 10^{-6}$ & $-2.1281788 * 10^{-6}$ & $1.5703716^{*} 10^{-6}$ \\
$\mathrm{~b}_{1}$ & 1.0001558 & 1.000203 & $9.9995809 * 10^{-1}$ \\
$\mathrm{~b}_{0}$ & $5.8185602 * 10^{-4}$ & $1.2782829 * 10^{-3}$ & $-1.6398909 * 10^{-3}$ \\
\hline
\end{tabular}

Then taking $\left\{\mathrm{b}_{0}(1500), \mathrm{b}_{0}(14 \mathrm{k}), \mathrm{b}_{0}(30 \mathrm{k})\right\},\left\{\mathrm{b}_{1}(1500), \mathrm{b}_{1}(14 \mathrm{k}), \mathrm{b}_{1}(30 \mathrm{k})\right\}$, and $\left\{b_{3}(1500), b_{3}(14 k), b_{3}(30 k)\right\}$ as the reference points, setting the frequency as the variable, each coefficient of the quadratic trinomial is fitting by the least-square method respectively, and the results are:

$$
b_{0}(f)=3.2284268 \times 10^{-4}+1.8520714 \times 10^{-7} \cdot f-8.3543865 \times 10^{-12} \cdot f^{2}
$$




$$
\begin{gathered}
b_{1}(f)=1.0001361-1.41437 \times 10^{-8} \cdot f-6.6925843 \times 10^{-13} \cdot f^{2} \\
b_{2}(f)=-1.5495608 \times 10^{-6}-1.6849151 \times 10^{-10} \cdot f+9.0829752 \times 10^{-15} \cdot f^{2}
\end{gathered}
$$

Assume that the calibration for the final output result is $\hat{y}$,then:

$$
\hat{y}=b_{2} \cdot x^{2}+b_{1} \cdot x+b_{0}
$$

By using the frequency of the input signal, coefficients $b_{2}, b_{1}, b_{0}$ can be calculated from Eqs. (17) (20). Finally, according to Eq. (20) , the final calibration value can be obtained. This method is used to calibrate the AC voltage signal range of $150 \mathrm{~V}$, and parts of the results are given in Table 4 .

In order to verify the validity of the method, the measurement data at 16 frequency points are selected in the $50 \mathrm{~Hz} \sim 30 \mathrm{kHz}$ wide band range. The error curve of the converter before and after the correction are plotted in Fig. 2 and Fig. 3 respectively. Error calibration accuracy is better than $0.018 \%$. The correction method is obviously better than the traditional least square method, which improves the accuracy of the broadband signal measurement.

Tab. 4 Comparison of data before and after correction

\begin{tabular}{ccccccccc}
\hline \multirow{2}{*}{$\begin{array}{c}\text { standard } \\
\text { value/V }\end{array}$} & \multicolumn{2}{c}{$3500 \mathrm{~Hz}$} & \multicolumn{2}{c}{$9000 \mathrm{~Hz}$} & \multicolumn{2}{c}{$20000 \mathrm{~Hz}$} & \multicolumn{2}{c}{$25000 \mathrm{~Hz}$} \\
\cline { 2 - 8 } & before & after & before & after & before & after & before & after \\
\hline 1.0000 & 0.979 & 1.001 & 0.979 & 1.002 & 0.959 & 1.001 & 0.949 & 1.000 \\
10.000 & 9.972 & 10.002 & 9.952 & 10.003 & 9.932 & 10.002 & 9.922 & 10.002 \\
80.000 & 79.875 & 80.002 & 79.825 & 80.003 & 79.695 & 80.005 & 79.685 & 80.005 \\
100.00 & 99.847 & 99.998 & 99.787 & 99.999 & 99.627 & 100.003 & 99.597 & 10.006 \\
120.00 & 119.803 & 119.993 & 119.705 & 119.993 & 119.503 & 120.000 & 119.503 & 120.007 \\
150.00 & 149.701 & 149.982 & 149.603 & 149.980 & 149.401 & 149.994 & 149.301 & 150.009 \\
\hline
\end{tabular}

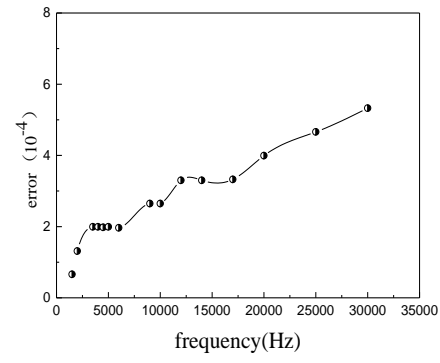

Fig. 2 Curve of the error frequency before correction

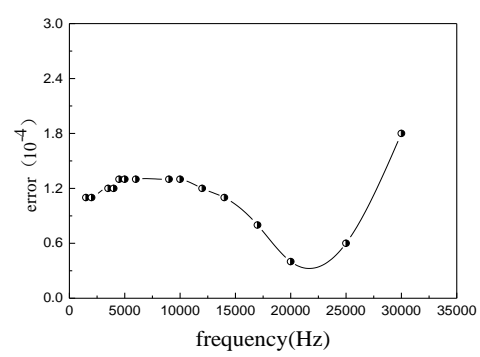

Fig. 3 Error frequency curve after correction 


\section{Conclusion}

In this paper, a novel method for frequency nonlinear error correction in wide frequency range is presented. This method combines the piecewise linear fitting method and the least square method, which can realize the nonlinear error calibration in the wide frequency band from $50 \mathrm{~Hz}$ to $30 \mathrm{kHz}$ wide band. The results show that the nonlinear error is reduced from $0.5 \%$ to $0.018 \%$. The new method greatly reduces the frequency nonlinearity error and improves the accuracy of the wideband signal measurement. The method is also suitable for the nonlinear error correction of other similar systems, and it has a certain reference value.

\section{References}

1. Xu W, Wang M, Cai J F, et al. Sparse Error Correction From Nonlinear Measurements With Applications in Bad Data Detection for Power Networks[J]. IEEE Transactions on Signal Processing, 2011, 61(24):61756187.

2. He Weiming, Song Xiaoqi,Gan Yi,Li Haolin, Jin Yuantou. Optimization of sensor calibration based on grey neural network modeling method [J]. Journal of instrument and meter, 2014,03:504-512(in Chinese).

3. Jiao L Y, Lei H Z. The application of improved genetic algorithm in fitting the spatial variogram[C]// Computer Science and Network Technology (ICCSNT), 2011 International Conference on. IEEE, 2011:1031-1036.

4. Chen Lei. Least squares support vector machine method for prediction[J]. Journal of Zhejiang University (Engineering Science Edition), 2011,06:1100-1103. (in Chinese).

5. Zhu Wu, Zhang Jiaming. Research on the automatic calibration method of the broadband RMS conversion curve[J]. Journal of instrument and meter, 2006,27(5):505-507. (in Chinese).

6. Chen Liangbo,Zheng Yaqing. Research on curve fitting based on least square method [J]. Journal of Wuxi Institute of Technology, ,2012,05:52-55 (in Chinese).

7. Tian Long,Liu Zongtian. Piecewise linear fitting by least square method[J]. computer science, ,2012,39(103):482-484. (in Chinese).

8. Kim M S, Kim W S, Kim Y G, et al. Power calibration system based on Josephson sampling voltmeter[J]. Cpem Digest, 2014:738-739. 